\title{
NIBS-driven brain plasticity
}

\author{
C. CHISARI', C. FANCIULLACCI', G. LAMOLA', B. ROSSI', L.G. COHEN² \\ ' Unit of Neurorehabilitation, University Hospital of Pisa, Italy; \\ ${ }^{2}$ Human Cortical Physiology and Neurorehabilitation Section, National Institute of Neurological \\ Disorders and Stroke, National Institutes of Health, Bethesda, Maryland, USA
}

\begin{abstract}
A B S T R A C T
Through plasticity the brain is able to change its function and to rearrange following injury or environmental changes. In recent years, it was shown that non-invasive brain stimulation (NIBS) techniques, especially transcranial magnetic stimulation (TMS) and transcranial direct current stimulation (tDCS) can contribute to understand how these plastic changes occur. Additionally, the literature suggests that TMS and tDCS may be used as interventional strategies to improve neurorehabilitation efforts and arguably recovery of motor function after brain lesions. This review focuses on the use of NIBS in experimental protocols for evaluation and modulation of brain plasticity, the factors contributing to the inter-individual variability of response, proposed mechanisms and difficulties in translating findings from small proof of principle studies through the pipeline to clinical practice.
\end{abstract}

Key words

Plasticity $\bullet$ Non-invasive brain stimulation $($ NIBS $) \bullet$ Neuromodulation $\bullet$ Transcranial magnetic stimulation $(T M S) \bullet$ Transcranial direct current stimulation (tDCS)

\section{Introduction}

Plasticity is the property of the nervous system to change its function and to rearrange following injury or environmental changes, through the establishment of new neural connections, the acquisition of new functions and the compensation for the damage (Cramer et al., 2011; Cheeran et al., 2009). It is important to emphasize that plasticity is not an occasional state of the nervous system: it is the normal ongoing state of the nervous system throughout the life span (Pascual-Leone et al., 2005).

Adaptive changes occurring during plasticity have been linked to the phenomenon of activity-dependent strengthening of synaptic transmission, called long-term potentiation (LTP) (Dimyan and Cohen, 2011). Instead, maladaptive plasticity corresponds to the reduction on behavioral capacity and may involve the reduction of strength of synapses called long-term depression (LTD) (Cohen et al., 1997). In recent years, an increasing interest has been directed towards evaluation and modification of plasticity in the cerebral cortex obtained by means of non-invasive brain stimulation (NIBS) techniques: the two forms of NIBS most widely used are transcranial magnetic stimulation (TMS) and transcranial direct current stimulation (tDCS). NIBS paradigms are applied in various ways (Dayan and Cohen, 2011; Dayan et al., 2013; Sandrini and Cohen, 2013): in experimental evaluative protocols as for LTP and LTD evaluation but also for therapeutic applications (Huang et al., 2009; Reis et al., 2008; Reis et al., 2008; Hummel et al., 2008; Ziemann et al., 2008).

Recent literature suggests that TMS and tDCS may be used as a possible technical adjuvant to customarily used neurorehabilitative treatments to enhance motor recovery (Liew et al., 2014). 
The following review focuses on the use of TMS and tDCS to understand the mechanisms of plasticity together with an analysis of the main NIBS therapeutic options for stroke rehabilitation, making at the same time an overview on the factors that may influence the effects of NIBS and on inter-individual variability of the response, finally trying to understand why these advanced techniques proved to be so useful but have not yet been transferred in common clinical practice.

Through the development of these concepts, this discussion reflects the great interest in the field of research that studies recovery of motor functions after neurological disorders such as stroke.

\section{Mechanisms of plasticity: the use of TMS}

TMS is a non-invasive tool that allows to study and to modulate the cortical excitability. The biophysical mechanisms induced by magnetic stimulation are still not completely understood. Given that the axons are the most effective conductors in the CNS, for their higher density of ion channels, the prevailing hypothesis is that they are preferentially affected by the TMS pulse, which may activate both inhibitory and excitatory neurons (Huerta et al., 2009). TMS may suppress neural signal or generate random neuronal noise; however, its effects have been suggested to be activity dependent, suppressing the most active neurons and changing the balance between excitation and inhibition (Pasley et al., 2009; Perini et al., 2012; Dayan et al., 2013).

Together with functional neuroimaging techniques like positron emission tomography (PET) and functional magnetic resonance imaging (fMRI), EEG and MEG, TMS has been used to improve our knowledge about brain plasticity underlying motor recovery (Schaechter, 2004).

\section{Single-pulse TMS}

Single-pulse TMS has been used to evaluate motor evoked potentials (MEPs) by stimulating the M1 area. The presence or absence of MEPs, together with other features like MEPs latency and amplitude, allows to study the integrity of the corticospinal tract. The corticospinal tract integrity, that can be also investigated through other techniques such as diffusion tensor imaging (DTI), showed great prog- nostic value in motor recovery of stroke patients, giving the opportunity to some authors (Stinear et al., 2007) to generate a flow-chart for prediction of motor recovery starting from the presence or absence of MEPs. In contrast with this, Swayne et al. (Swayne et al., 2008) showed a poor correlation between MEP amplitude and clinical deficit at 6 months after stroke. They hypothesized that the recovery of motor function is more dependent on the reorganization of alternative cortical networks than on the function of the original corticospinal pathways spared by the ischemic lesions. Therefore, LTP-like plasticity in the acute phase might be the expression of the potential of these alternative cortical networks.

Single-pulse TMS was also used to show that a specific area in the parietal cortex mediates spatial orienting during distinct time periods after the onset of the behavioral event, suggesting that fast and slow visual pathways are necessary to orient spatial attention. Furthermore, single-pulse TMS has contributed to the understanding of the mechanisms of motor learning (Bütefisch et al., 2004): use-dependent encoding of a motor memory can be enhanced by synchronous TMS applied to the motor cortex engaged in the motor training task.

\section{Double-pulse TMS}

A different type of TMS, called paired-pulse TMS (ppTMS), has significantly advanced our understanding of mechanisms underlying the excitability of the motor cortex. Single-region ppTMS is typically limited to M1 and involves the application of both a subthreshold conditioning stimulus and suprathreshold test stimulus (inducing a measurable MEP) to the same region. The precise interstimulus interval between the conditioning and test stimuli can result in intracortical inhibitory (with an interstimulus interval $<5 \mathrm{~ms}$ ) or facilitory (if the conditioning stimulus precedes the test stimulus by latencies between 6 and $25 \mathrm{~ms}$, or if the conditioning stimulus succeeds the test stimulus at $1.5-\mathrm{ms}$ intervals between 1 and $4.5 \mathrm{~ms}$ ) effects on corticospinal output (Ziemmann et al., 1996; Hallett, 2007).

ppTMS can be used to study functional interactions within a single brain region or between two connected brain areas (Reis et al., 2008), but also to investigate interactions between two spatially distinct brain regions. In fact, cortico-cortical connectivity 
can be assessed between homologous regions of M1 of each hemisphere (Di Lazzaro, 1999), or between M1 and other regions like premotor cortex, dorsolateral prefrontal cortex, posterior parietal cortex and so on (Koch et al., 2007; Daskalakis et al., 2008; Buch et al., 2010). The study of these dynamics in chronic stroke patients may bring up interesting revelations about the causal relationships between altered cortico-cortical interactions and behavioral deficits, allowing to investigate the role of prefrontal, frontal and parietal inputs on M1 corticospinal output in motor behavioral contexts such as prehension, action selection and action reprogramming (Liew et al., 2014).

\section{rTMS and TBS}

Besides, the use of particular protocols of repetitive transcranial magnetic stimulation (rTMS) enabled to produce a prolonged modification in cortical excitability with LTP- and LTD-like changes.

rTMS can be applied using protocols in which stimulation and task performance are dissociated in time. The induced effects outlast the period of stimulation, giving insight into the role of the specific stimulated brain regions in plasticity and behavior. In the motor system, low-frequency $(1 \mathrm{~Hz})$ rTMS inhibits cortical excitability, creating a transient "virtual lesion" (Chen et al., 1997). Instead, high-frequency $(5-20 \mathrm{~Hz})$ rTMS produces an increase in cortical excitability (Pascual-Leone et al., 1994), which can facilitate motor sequence learning (Kim et al., 2004), though the effects may vary (Agostino et al., 2007). The molecular mechanism of action of rTMS has been recently studied, focusing on the effect on N-methyl-D-aspartate (NMDA) transmission and the interaction on Brain-derived neurotrophic factor (BDNF) and its receptor, tyrosine kinase receptor B (TrkB) (Wang et al., 2011). In normal human subjects, 5-day rTMS treatment over motor cortex decreases resting motor threshold correlating with heightened BDNF-TrkB signaling accompanied by an increased association between the activated TrkB and NMDA receptor (NMDAR), with a resultant BDNF-TrkB-NMDAR functioning facilitation in both cortex and lymphocytes.

A way to induce longer-lasting effects than conventional rTMS paradigms (Dayan et al., 2013) is thetaburst stimulation (TBS), which involves the application of a burst of three $50-\mathrm{Hz}$ pulses in trains repeated at 200-ms intervals. Continuous TBS (cTBS) consists of the application of burst trains for 20-40 s and has an inhibitory effect on corticospinal excitability. Instead, for intermittent TBS (iTBS), burst trains with a duration of $2 \mathrm{~s}$ are applied over a total of $190 \mathrm{~s}$, with the trains repeating every $10 \mathrm{~s}$ (Huang et al., 2005). iTBS can induce LTP-like changes in the stimulated hemisphere and LTD-like changes in the opposite hemisphere. Di Lazzaro et al. (2008) evaluated LTPand LTD-like changes in excitability of the affected and unaffected hemispheres produced by iTBS in 17 acute ischemic stroke, finding that functional recovery is directly correlated with LTP-like changes in affected hemisphere and LTD-like changes in unaffected hemisphere and inversely correlated with the baseline excitability of unaffected hemisphere. Back to the molecular mechanisms, Huang et al. (2007) provided pharmacological evidence that the effects of plasticity like after-effects in the motor cortex due to iTBS and cTBS rely on NMDA receptors.

\section{Paired associative stimulation}

Paired associative stimulation (PAS) is another stimulation protocol widely used for demonstrating LTP-like and LTD-like phenomena. PAS takes advantage of the principles of associative plasticity by repeatedly coupling a low-frequency peripheral stimulation from the median nerve with a cortical TMS pulse applied over contralateral motor cortex, with an inter-stimulus interval (ISI) of $10-25 \mathrm{~ms}$ (Stefan et al., 2000). An ISI of $10 \mathrm{~ms}$ induces a depression of TMS-evoked MEPs, while enhancement of cortical excitability is consequent to the use of $25 \mathrm{~ms}$ of ISI, with effects of at least 1 hour of duration and resembling LTP-like and LTD-like mechanisms. Protocols using PAS are particularly relevant because they demonstrate some characteristics of spike timing-dependent plasticity (Wolters et al., 2003): the order and precise temporal interval between presynaptic and postsynaptic spikes determine the sign and magnitude of LTP-like or LTDlike synaptic changes.

\section{Mechanisms of plasticity: the use of tES}

Transcranial electric stimulation (tES) is a method that has attracted significant attention because its application is thought to induce neuromodulation, as shown by improvements in behavioral and cogni- 
tive performance in normal and pathological subjects (Miniussi et al., 2011). Different types of tES are differentiated by specific modalities of current discharge (e.g., direct versus alternating) that might have different neuromodulatory effects on cortical networks.

\section{tDCS}

The growing interest in NIBS generated by TMS led to the revitalization of tDCS, a technique whose effects were already systematically analyzed in animal models in the 1960s (Albert, 1966a; Albert, 1966b) and even studied in humans (Elbert et al., 1981). In 2000 Nitsche has demonstrated that tDCS induces cortical excitability changes in the human motor cortex (Nitsche et al., 2000). tDCS offers the possibility to change cortical excitability in a polarity-specific manner (anodal versus cathodal) and this can be achieved by the application of electrodes with different polarity to different locations on the surface of the skull to excite the underlying neural tissue (Utz et al., 2010). tDCS effects are most likely induced by membrane polarization, altering the firing rates of neurons (Fritsch et al., 2010). Anodal tDCS induces depolarization, while cathodal tDCS induces hyperpolarization, so that anodal stimulation produces excitation and cathodal stimulation produces inhibition (Liebetanz et al., 2002). Some studies suggested that the immediate effects of tDCS on corticospinal excitability primarily depend on subthreshold resting membrane potential changes, whereas aftereffects of tDCS are due to shifts in intracortical inhibition and facilitation, and interactions with facilitatory corticospinal waves (Nitsche et al., 2005). tDCS effects have been shown in a wide range of processes, spanning motor and sensory to cognitive functions (Utz, et al., 2010).

\section{tRNS and tACS}

Transcranial random noise stimulation (tRNS) is a repetitive alternating current of different frequencies applied in a random mode to the cortex through the scalp (Fertonani et al., 2011). Terney et al. (Terney et al., 2008) reported that tRNS improved the performance of implicit motor learning tasks and increased motor cortex excitability. Therefore, we can presume that tRNS can change cortical excitability by inducing depolarization.

Also transcranial alternating current stimulation (tACS) has been proposed as a tool for investigat- ing cortical mechanisms (Antal et al., 2008). tACS refers to an electrical stimulation where current is not constant but alternates between the anode and the cathode (switching polarity) with a sinusoidal waveform, interfering with ongoing oscillations in the brain (Marshall et al., 2006). The predominant hypothesis of tACS action is that alternating fields can increase or decrease power of oscillatory rhythms in the brain in a frequency-dependent manner by synchronizing or desynchronizing neuronal networks (Reato et al., 2013). If applied long enough, tACS may cause neuroplastic effects. When applied in phase in the theta range it may improve cognition (Montez, 2009). Alpha rhythms could improve motor performance, whereas beta intrusion may deteriorate them (Feurra et al., 2011; Neuling et al., 2012). Gamma intrusion can possibly interfere with attention (Bartos et al., 2007). Stimulation in the "ripple" range induces intensity dependent inhibition or excitation in M1 most likely by entrainment of neuronal networks, whereas stimulation in the low $\mathrm{kHz}$ range induces excitation by neuronal membrane interference (Antal and Paulus, 2013).

All these techniques have proved capable of produce neuroplastic effects if applied with appropriate parameters in terms of frequency and intensity (Paulus, 2011), although further studies would be useful for a complete understanding of the dynamics underlying plasticity.

\section{NIBS therapy and the mechanisms of motor recovery}

\section{NIBS to enhance motor recovery}

In addition to their use to evaluate neuroplasticity, NIBS techniques are tested as interventional options for stroke rehabilitation with the aim to safely augment neural plasticity and improve motor function. In part, their use is based on the interhemispheric competition model, a model based on the concept that motor deficits in stroke patients relate to reduced output from the affected hemisphere and excessive interhemispheric inhibition from the unaffected hemisphere to the affected hemisphere (Kinsbourne et al., 1977; Kinsbourne et al., 1980; Murase et al., 2004; Takeuchi et al., 2005). It has been proposed that using NIBS, improvement in motor deficits can be achieved by increasing the excitability of the 
affected hemisphere or decreasing the excitability of the unaffected hemisphere (Ward and Cohen, 2004; Nowak et al., 2009). This model has been recently brought into question by Di Lazzaro et al. (2013): they used inhibitory TBS of affected hemisphere in chronic stroke patients to verify if this intervention had the potential to enhance recovery. Results showed clinical improvements for up to 3 months post-treatment, suggesting the possibility to design protocols of inhibition of affected hemisphere for chronic stroke patients. Overall, it is conceivable that upregulation and downregulation of activity in the affected hemisphere may partially contribute depending on different factors like magnitude of baseline motor function (Fridman et al., 2004).

Excitability enhancement in the motor cortex appears to be required for motor learning (PascualLeone et al., 1998, Muelbacher et al., 2002; Reis et al., 2009; Censor et al., 2010; Schambra et al., 2011). Therefore, NIBS can facilitate motor learning and induce motor recovery by directly or indirectly increasing the excitability in the ipsilesional motor cortex. In fact, compared to motor training or rTMS alone, pairing motor training with rTMS results in prolonged performance improvements and functional neural plasticity in the ipsilesional motor cortex (Nowak et al., 2009; Takeuchi et al., 2009).

Studies using functional neuroimaging have found that cerebral blood flow of the ipsilesional motor cortex is increased after inhibitory rTMS over the unaffected hemisphere and excitatory rTMS over the affected hemisphere (Conchou et al., 2009; Ameli et al., 2009). These NIBS-induced metabolic changes may also promote neural plasticity and motor recovery after stroke (Conchou et al., 2009). Moreover, excitatory NIBS over the affected hemisphere can induce LTP-like changes in the affected hemisphere and promote motor recovery after stroke (Di Lazzaro et al., 2010). Therefore, NIBS may resolve impairment of experience-dependent plasticity in the affected hemisphere after stroke (Carmichael, 2006; Di Filippo et al., 2008; Takeuchi and Izumi, 2012b). These findings suggest that artificially modulating the cortical excitability by NIBS may induce a more suitable environment for neural plasticity (Takeuchi and Izumi, 2012a).

In addition to excitability modulation in the ipsilesional motor cortex, NIBS may modulate the neural network in both hemispheres to induce motor recov- ery. In this way, fMRI and EEG studies may show the effects of NIBS therapy for stroke on the motor cortical network.

In this regard, a neuroimaging study conducted with fMRI revealed that inhibitory rTMS over the unaffected hemisphere reduced the pathological hyperactivity in the primary and non-primary motor cortices in the unaffected hemisphere (Nowak et al., 2008). Excitatory rTMS over the affected hemisphere has been shown to reduce neural activity in the contralesional motor cortex, in addition to facilitation of the ipsilesional motor cortex (Ameli et al., 2009). Moreover, inhibitory rTMS over the unaffected hemisphere reduced the connectivity of both hemispheres and enhanced coupling between the primary and nonprimary motor cortices in the affected hemisphere (Grefkes et al., 2010; Takeuchi and Ikoma, 2010). Enhanced excitability in the unaffected hemisphere inhibits the affected hemisphere via excessive interhemispheric inhibition and weakens motor function of the paretic side (Murase et al., 2004). Although the change in neural coupling after excitatory NIBS remains still unclear, normalized excitability of both hemispheres and reconstruction of effective connectivity between the primary and nonprimary motor cortices in the affected hemisphere after NIBS may contribute to motor recovery in stroke patients (Takeuchi and Izumi, 2012).

\section{The role of NIBS as neuroprotective option}

The underlying mechanisms of rTMS in stroke recovery remain partially unclear. Yoon et al. (2011) evaluated those mechanisms by assessing proteins associated with neural plasticity and anti-apoptosis in the peri-lesional area in subacute cerebral ischemic rat model. They suggested that rTMS therapeutic effect on functional recovery may be associated with an anti-apoptotic mechanism in the peri-ischemic area rather than with neural plasticity. This may also suggest an intriguing neuroprotective role of NIBS.

Beyond the interhemispheric competition model: the role of unaffected hemisphere

As mentioned above, the interhemispheric competition model is very attractive but may be too simplistic to explain entirely brain reorganization after the stroke event. In fact, the role of the unaffected hemisphere for the recovery of motor function 
after stroke is still controversial and it is not certain that his hyper activation is only an expression of maladaptive plasticity. Some author suggested that unaffected hemisphere could in some instances even contribute to motor recovery such as in language functions. Cao et al. (1999) investigated with fMRI cortical language activation in patients recovering from aphasia after a single left-hemisphere ischemic infarct. They found that two factors contributed to recovery: restitution of damaged left hemisphere language networks and activation of compensated (or recruited) areas in the right hemisphere (the results also confirmed that after focal left-hemisphere damage, a bilaterally reorganized language network works more effectively than a right-predominant network, as already suggested in a previous work by Karbe et al.).

More recently, Riecker et al. (2010) used fMRI to study task-related activation in unaffected hemisphere during finger movements in subcortical stroke patients compared to healthy controls. A linear increase of the hemodynamic response with higher tapping frequencies in unaffected side (in premotor and primary sensorimotor cortex) was only seen in stroke patients. These results support the model of an enhanced bihemispheric recruitment of preexisting motor representations in patients after subcortical stroke. Since all patients had excellent motor recovery unaffected primary sensorimotor cortex activation appears to be efficient and resembles the widespread, bilateral activation observed in healthy subjects performing complex movements, instead of reflecting maladaptive plasticity.

\section{Influence of neurophysiological and clinical factors on the effects of NIBS}

The influence of the activation state of the brain The pattern of neural network activation in both hemispheres has important influences on the effect of NIBS therapy for motor stroke patients. It was reported that good responders for inhibitory NIBS over the unaffected hemisphere have increased fMRI activity in the contralesional dorsal premotor cortex and contralesional parietal operculum before stimulation (Nowak et al., 2008). On the other hand, a previous study reported that ipsilesional motor cortex excitability in good responders for excitatory
NIBS over the affected hemisphere is easily facilitated by moving the paretic hand before stimulation (Ameli et al., 2009). Therefore, it seems to be important to develop predictors of NIBS response at an individual patient level.

Nowadays it is known that the neural impact of a NIBS is not determined only by the properties of the stimulus but also on the activation state of the brain. In fact, the response depends on how excitable the cortex is at the time the stimulus is applied: if many neurons are close to motor threshold, then the more of them are recruited by the pulse than at rest. This "state-dependency" is a general feature of cortical neural processing and it plays an important role on the efficacy of TMS protocols (Silvanto \& PasqualLeone, 2008).

In accordance with the view of state-dependent stimulation effects, a new method called TMS adaptation (TMSA) has been introduced to increase the functional resolution of TMS (Dayan et al., 2013). An adapting stimulus, presented for a long time (usually 40-60 s), is used to induce habituation in a subset of cells that encode particular stimulus attributes, therefore making them a selective target for TMS (Sandrini et al., 2011). TMSA predicts that TMS improves processing of attributes that are adapted, whereas it decreases performance for non-adapted attributes. Nevertheless, the underlying mechanism of TMS and TMSA are still debated. A recent study manipulated the brain state using contrast adaptation, a decrease in visual contrast sensitivity produced by repeated exposure to highcontrast stimuli (Perini et al., 2012). TMS impaired perception when the visual cortex was not adapted but facilitated perception after adaptation. It has been proposed that TMS affects excitatory and inhibitory neural populations differentially and that TMS has an activity-dependent suppressive effect on the inhibitory populations.

Therefore, since the TMS after effects are extremely variable, future strategies should taking into account the closed loop stimulation which would consider the ongoing electrical cerebral activity to get the after effects more predictable.

\section{The influence of clinical factors}

Actually the clinical characteristics that make patients more responsive to NIBS treatment remain unclear. First, it is necessary to consider the char- 
acteristics of stroke. From the literature, it has been proposed that NIBS therapy may be more effective in acute stages (Takeuchi and Izumi, 2012). Therefore, early modulation of cortical excitability may facilitate motor recovery and prevent development of maladaptive neural plasticity by rebalancing interhemispheric communication and normalizing neural activity within the motor areas of both hemispheres. Although it has been reported that both inhibitory and excitatory NIBS may contribute to motor recovery in acute stroke patients (Khedr et al., 2009; Di Lazzaro et al., 2010; Khedr et al., 2010), a recent study showed that inhibitory NIBS does not facilitate motor recovery in this phase of the disease (Seniów et al., 2012).

The lesion size may influence the NIBS effects. Studies on excitatory NIBS over the affected hemisphere reported that no difference occurs in motor improvement between patients with subcortical and cortical stroke lesions (Khedr et al., 2005; Kim et al., 2006). However, a study showed that excitatory NIBS over the affected hemisphere improves dexterity of the paretic hand in subcortical stroke patients but not in some cortical-subcortical stroke patients (Ameli et al., 2009). Excitatory rTMS over the affected hemisphere reduced neural activity of the contralesional motor cortex in patients with subcortical stroke but caused widespread bilateral recruitment of primary and nonprimary motor areas in patients with cortical stroke (Ameli et al., 2009). Thus the proposal that NIBS interventions might be less effective for cortical stroke patients (Takeuchi and Izumi, 2012).

\section{Inter-individual variability response to NIBS}

Stimulation-induced behavioral, physiological and therapeutic effects are not uniform and tend to considerably vary among individuals. A number of reasons have been suggested for this variability, such as time of day (Ridding and Ziemann, 2010), anatomical aspects as cortical thickness (Conde et al., 2012), coil orientation (Talelli et al., 2007), genetic variation (Cheeran et al., 2008). Indeed, several factors have been shown to modulate the magnitude of NIBS effects, including variation in brain and skull morphology, local brain oscillations, age, physi- cal fitness and sex (Ridding and Ziemann, 2010). Moreover, polymorphisms in genes related to dopamine (Plewnia et al., 2013) and BDNF (Cheeran et al., 2008; Fritsch et al., 2010) explain some of the variation in NIBS-induced plasticity and have thus pointed to mediation by these mechanisms (Dayan et al., 2013).

However very recent studies underlined that the subjects that respond to NIBS protocol show an important modification in cortical excitability, in particular their MEP amplitude increases by $300 \%$ at $60 \mathrm{~min}$ post stimulation, suggesting that individual response could to some extent be predicted by careful evaluation of effects on cortical excitability, also an important factor that could influence safety of NIBS protocols (Lopez-Alonso et al., 2014).

\section{Conclusions}

Understanding how people recover after stroke represents one of the biggest enigmas in contemporary neuroscience and to find a way to investigate the mechanisms of recovery is becoming always more important.

NIBS techniques are non-invasive tools that allow the study and modulation of cortical excitability. By means of NIBS we are reaching a greater understanding about the ability of nervous system to change its function and to rearrange after stroke. Certainly, it is difficult to compare results from different studies. There is a lack of homogeneity among individuals in stimulation-induced behavioral, physiological and therapeutic experimental conditions. Similar heterogeneity is present in the patients' lesion locations, genotype information, parameters of stimulation and state-dependency. A further problem consists in poor standardization together with other limitations in the methods used in research protocols (number of patients, stimulation parameters, degree of preparation of professionals carrying out the stimulation protocols, type of clinical evaluations, descriptions of the control conventional therapy), and more importantly blinding so that additional efforts are required to achieve a greater uniformity and accuracy of protocols.

A first attempt to draft guidelines on the therapeutic use of rTMS was recently published (Lefaucheur et al., 2014), classifying evidence-based notions in dif- 
ferent levels of recommendations and combining a specific type of rTMS to a specific type of disorder. However, more work is necessary to determine which interventional protocols can optimally facilitate motor recovery or which patients are more likely to be benefited. One of the unsolved questions is the concept of NIBS combined with motor behaviour and particularly rehabilitation. At the present it is still unknown if TMS or tDCS should be delivered before, during or after the motor task. The timing and the combination of neuromodulation and motor behaviour could represent one of the key points of future research.

\section{References}

Agostino R., Iezzi E., Dinapoli L., Gilio F., Conte A., Mari F., Berardelli A. Effects of $5 \mathrm{~Hz}$ subthreshold magnetic stimulation of primary motor cortex on fast finger movements in normal subjects. Exp. Brain Res., 180: 105-111, 2007.

Albert D.J. The effect of spreading depression on the consolidation of learning. Neuropsychologia, 4: 49-64, 1966a.

Albert D.J. The effects of polarizing currents on the consolidation of learning. Neuropsychologia, 4: 65-77, 1966b.

Ameli M., Grefkes C., Kemper F, Riegg F.P., Rehme A.K., Karbe H., Fink G.R., Nowak D.A. Differential effects of high-frequency repetitive transcranial magnetic stimulation over ipsilesional primary motor cortex in cortical and subcortical middle cerebral artery stroke. Ann. Neurol., 66: 298-309, 2009.

Antal A., Boros K., Poreisz C., Chaieb L., Terney D., Paulus W. Comparatively weak after-effects of transcranial alternating current stimulation (tACS) on cortical excitability in humans. Brain Stimul., 1: 97-105, 2008.

Antal A. and Paulus W. Transcranial alternating current stimulation (tACS). Front. Hum. Neurosci., 7: $317,2013$.

Bartos M., Vida I., Jonas P. Synaptic mechanisms of synchronized gamma oscillations in inhibitory interneuron networks. Nat. Rev. Neurosci., 8: 45-56, 2007.

Bikson M., Rahman A., Datta A. Computational models of transcranial direct current stimulation. Clin. EEG Neurosci., 43: 176-183, 2012.

Bütefisch C.M., Khurana V., Kopylev L., Cohen L.G. Enhancing encoding of a motor memory in the primary motor cortex by cortical stimulation. $J$. Neurophysiol., 91:, 2110-2116, 2004.

Buch E.R., Mars R.B., Boorman E.D., Rushworth M.F. A network centered on ventral premotor cortex exerts both facilitatory and inhibitory control over primary motor cortex during action reprogramming. J. Neurosci., 30: 1395-1401, 2010.

Cao Y., Vikingstad E.M., George K.P., Johnson A.F., Welch K.M.A. Cortical language activation in stroke patients recovering from aphasia with functional MRI. Stroke, 30: 2331-2340, 1999.

Carmichael S.T. Cellular and molecular mechanisms of neural repair after stroke: making waves. Ann. Neurol., 59: 735-742, 2006.

Censor N., Dimyan M.A., Cohen L.G. Modification of existing human motor memories is enabled by primary cortical processing during memory reactivation. Curr. Biol., 20: 1545-1549, 2010.

Cheeran B., Cohen L., Dobkin B., Ford G., Greenwood R., Howard D., Husain M., Macleod M., Nudo R., Rothwell J., Rudd A., Teo J., Ward N., Wolf S. The future of restorative neurosciences in stroke: driving the translational research pipeline from basic science to rehabilitation of people after stroke. Neurorehabil. Neural Repair, 23: 97-107, 2009.

Cheeran B., Talelli P., Mori F., Koch G., Suppa A., Edwards M., Houlden H., Bhatia K., Greenwood R., Rothwell J.C. A common polymorphism in the brain-derived neurotrophic factor gene (BDNF) modulates human cortical plasticity and the response to rTMS. J. Physiol., 586 (Pt 23): $5717 \mathrm{e} 25,2008$.

Chen R., Classen J., Gerloff C., Celnik P., Wassermann E.M., Hallett M., Cohen L.G. Depression of motor cortex excitability by low-frequency transcranial magnetic stimulation. Neurology, 48: 1398-1403, 1997.

Cohen, L.G., Celnik P., Pascual-Leone A., Corwell B., Falz L., Dambrosia J., Honda M., Sadato N., Gerloff C., Catala M.D., Hallett M. Functional relevance of cross-modal plasticity in blind humans. Nature, 389: 180-183, 1997.

Conde V., Vollmann H., Sehm B., Taubert M., Villringer A., Ragert P. Cortical thickness in primary sensorimotor cortex influences the effectiveness of paired associative stimulation. Neuroimage, 60: $864 \mathrm{e} 70,2012$.

Conchou F., Loubinoux I., Castel-Lacanal E, Le Tinnier A., Gerdelat-Mas A., Faure-Marie N., Gros H., Thalamas C., Calvas F., Berry I., Chollet F., Simonetta Moreau M. Neural substrates of low- 
frequency repetitive transcranial magnetic stimulation during movement in healthy subjects and acute stroke patients. A PET study. Hum. Brain Mapp., 30: 2542-2557, 2009.

Cramer S.C., Sur M., Dobkin B.H., O'Brien C., Sanger T.D., Trojanowski J.Q., Rumsey J.M., Hicks R., Cameron J., Chen D., Chen W.G., Cohen L.G., deCharms C., Duffy C.J., Eden G.F., Fetz E.E., Filart R., Freund M., Grant S.J., Haber S., Kalivas P.W., Kolb B., Kramer A.F., Lynch M., Mayberg H.S., McQuillen P.S., Nitkin R., PascualLeone A., Reuter-Lorenz P., Schiff N., Sharma A., Shekim L., Stryker M., Sullivan E.V., Vinogradov S. Harnessing neuroplasticity for clinical applications. Brain, 134 (Pt 6): 1591-1609, 2011.

Daskalakis Z.J., Farzan F., Barr M.S., Maller J.J., Chen R., Fitzgerald P.B. Long-interval cortical inhibition from the dorsolateral prefrontal cortex: a TMS-EEG study. Neuropsychopharmacology, 33: 2860-2869, 2008.

Dayan E. and Cohen L.G. Neuroplasticity subserving motor skill learning. Neuron, 72: 443-454, 2011.

Dayan E., Censor N., Buch E.R., Sandrini M., Cohen L.G. Noninvasive brain stimulation: from physiology to network dynamics and back. Nat. Neurosci., 16: 838-844, 2013.

Di Filippo M., Tozzi A., Costa C., Belcastro V., Tantucci M., Picconi B., Calabresi P. Plasticity and repair in the post-ischemic brain. Neuropharmacology, 55: 353-362, 2008.

Di Lazzaro V., Oliviero A., Profice P., Insola A., Mazzone P., Tonali P., Rothwell J.C. Direct demonstration of interhemispheric inhibition of the human motor cortex produced by transcranial magnetic stimulation. Exp. Brain Res., 124: 520-524, 1999.

Di Lazzaro V., Profice P., Pilato F., Capone F., Ranieri F., Pasqualetti P., Colosimo C., Pravatà E., Cianfoni A., Dileone M. Motor cortex plasticity predicts recovery in acute stroke. Cereb. Cortex, 20: 1523-1528, 2010.

Di Lazzaro V., Rothwell J.C., Talelli P., Capone F., Ranieri F., Wallace A.C., Dileone M. Inhibitory theta burst stimulation of affected hemisphere in chronic stroke: A proof of principle, sham-controlled study. Neurosci. Lett., 553: 148-152, 2013.

Dimyan M.A. and Cohen L.G. Neuroplasticity in the context of motor rehabilitation after stroke. Nat. Rev. Neurol., 7: 76-85, 2011.

Elbert T., Lutzenberg W., Rockstroh B., Birbaumer $\mathrm{N}$. The influence of low-level transcortical DC-currents on response speed in humans. Intl. $J$. Neurosci., 14: 101-114, 1981.
Fertonani A., Pirulli C., Miniussi C. Random noise stimulation improves neuroplasticity in perceptual learning. J. Neurosci., 31: 15416e23, 2011.

Feurra M., Bianco G., Santarnecchi E., Del Testa M., Rossi A., Rossi S. Frequency-dependent tuningofthe human motor system induced by transcranial oscillatory potentials. J. Neurosci., 31: 12165-12170, 2011.

Fridman E.A., Hanakawa T., Chung M., Hummel F., Leiguarda R.C., Cohen L.G. Reorganization of the human ipsilesional premotor cortex after stroke. Brain, 127 (Pt 4): 747-758, 2004.

Fritsch B., Reis J., Martinowich K., Schambra H.M., Ji Y., Cohen L.G., Lu B. Direct current stimulation promotes BDNF-dependent synaptic plasticity: potential implications for motor learning. Neuron, 66: 198-204, 2010.

Grefkes C., Nowak D.A., Wang L.E., Dafotakis M., Eickhoff S.B., Fink G.R. Modulating cortical connectivity in stroke patients by rTMS assessed with fMRI and dynamic causal modeling. Neurolmage, 50: 233-242, 2010.

Hallett M. Transcranial magnetic stimulation: a primer. Neuron, 55: 187-199, 2007.

Huang Y.-Z., Edwards M.J., Rounis E., Bhatia K.P., Rothwell J.C. Theta burst stimulation of the human motor cortex. Neuron, 45: 201-206, 2005.

Huerta P.T. and Volpe B.T. Transcranial magnetic stimulation, synaptic plasticity and network oscillations. J. Neuroeng. Rehabil., 6: 7, 2009.

Karbe H., Thiel A., Weber-Luxenburger G., Herholz K., Kessler J., Heiss W.D. Brain plasticity in poststroke aphasia: what is the contribution of the right hemisphere? Brain Lang., 64: 215-230, 1998.

Khedr E.M., Abdel-Fadeil M.R., Farghali A., Qaid M. Role of 1 and $3 \mathrm{~Hz}$ repetitive transcranial magnetic stimulation onmotor function recovery after acute ischaemic stroke. Eur. J. Neurol., 16: 1323-1330, 2009.

Khedr E.M., Etraby A.E., Hemeda M., Nasef A.M., Razek A.A.M. Long-term effect of repetitive transcranial magnetic stimulation on motor function recovery after acute ischemic stroke. Acta Neurol. Scand., 121: 30-37, 2010.

Kim Y.H., You S.H., Ko M.H., Park J.W., Lee K.H., Jang S.H., Yoo W.K., Hallett M. Repetitive transcranial magnetic stimulation-induced corticomotor excitability and associated motor skill acquisition in chronic stroke. Stroke, 37: 1471-1476, 2006.

Kim Y.H., Park J.W., Ko M.H., Jang S.H., Lee P.K. Facilitative effect of high frequency subthreshold 
repetitive transcranial magnetic stimulation on complex sequential motor learning in humans. Neurosci. Lett. 367: 181-185, 2004.

Kinsbourne M. Hemi-neglect and hemisphere rivalry. Adv. Neurol., 18: 41-49, 1977.

Kinsbourne M. Dichotic imbalance due to isolated hemisphere occlusion or directional rivalry? Brain Lang., 11: 221-224, 1980.

Koch G., Fernandez Del Olmo M., Cheeran B., Ruge D., Schippling S., Caltagirone C., Rothwell J.C. Focal stimulation of the posterior parietal cortex increases the excitability of the ipsilateral motor cortex. J. Neurosci., 27: 6815-6822, 2007.

Lefaucheur J.P., André-Obadia N., Antal A., Ayache S.S., Baeken C., Benninger D.H., Garcia-Larrea L. Evidence-based guidelines on the therapeutic use of repetitive transcranial magnetic stimulation (rTMS). Clin. Neurophysiol., 125: 2150-2206, 2014.

Liebetanz D., Nitsche M.A., Tergau F., Paulus W. Pharmacological approach to the mechanisms of transcranial DC-stimulation-induced after-effects of human motor cortex excitability. Brain, 125 (Pt 10): $2238 \mathrm{e} 47,2002$

Liew S.L., Santarnecchi E., Buch E., Cohen L. Noninvasive brain stimulation in neurorehabilitation: Local and distant effects for motor recovery. Front. Hum. Neurosci., 8: 378, 2014.

Marshall L., Helgadottir H., Molle M., Born J. Boosting slow oscillations during sleep potentiates memory. Nature, 444: 610-613, 2006.

Miniussi C. and Vallar G. Brain stimulation and behavioural cognitive rehabilitation: a new tool for neurorehabilitation? Neuropsychol. Rehabil., 21: 553e9, 2011.

Montez T., Poil S.S., Jones B.F., Manshanden I., Verbunt J.P., vanDijk B.W., Brussaardc A.B., van Ooyenc A., Stamb C.J., Scheltense P., LinkenkaerHansen K. Altered temporal correlations in parietal alpha and prefrontal theta oscillations in earlystage Alzheimer disease. Proc. Natl. Acad. Sci. USA, 106: 1614-1619, 2009.

Muellbacher W., Zlemann U., Wissel J., Dang N., Kofler M., Facchini S., Boroojerdi B., Poewe W., Hallett M. Early consolidation in human primary motor cortex. Nature, 415: 640-644, 2002.

Murase N., Duque J., Mazzocchio R., Cohen L.G. Influence of interhemispheric interactions on motor function in chronic stroke. Ann. Neurol., 55: 400-409, 2004.

Nitsche M.A. and Paulus W. Excitability changes induced in the human motor cortex by weak transcranial direct current stimulation. J. Physiol. (Lond.), 527: 633-639, 2000.

Nitsche M.A., Seeber A., Frommann K., Klein C.C., Rochford C., Nitsche M.S., Fricke K., Liebetanz D., Lang N., Antal A., Paulus W., Tergau F. Modulating parameters of excitability during and after transcranial direct current stimulation of the human motor cortex. J. Physiol. (Lond.), 568: 291303, 2005.

Nitsche M.A. and Paulus W. Transcranial direct current stimulation. Update 2011. Restor. Neurol. Neurosci., 29: 463e92, 2011.

Neuling T., Rach S., Wagner S., Wolters C.H., Herrmann C.S. Good vibrations: oscillatory phase shapes perception. Neuroimage, 63: 771-778, 2012.

Nowak D.A., Grefkes C., Dafotakis M., et al. Effects of low-frequency repetitive transcranial magnetic stimulation of the contralesional primary motor cortex on movement kinematics and neural activity in subcortical stroke. Arch. Neurol., 65: 741-747, 2008.

Pascual-Leone A., Tarazona F., Keenan J., Tormos J.M., Hamilton R., Catala M.D. Transcranial magnetic stimulation and neuroplasticity. Neuropsychol., 37: 207-217, 1998.

Pascual-Leone A., Grafman J., Hallett M. Modulation of cortical motor output maps during development of implicit and explicit knowledge. Science, 263: 1287-1289, 1994.

Pascual-Leone A., Amedi A., Fregni F., Merabet L.B. The plastic human brain cortex. Annu. Rev. Neurosci., 28: 377-401, 2005.

Pasley B.N., Allen E.A., Freeman R.D. Statedependent variability of neuronal responses to transcranial magnetic stimulation of the visual cortex. Neuron, 62: 291-303, 2009.

Paulus W. Transcranial electrical stimulation (tEStDCS; tRNS, tACS) methods. Neuropsychol. Rehabil., 21: 602-617, 2011.

Perini F., Cattaneo L., Carrasco M., Schwarzbach J.V. Occipital transcranial magnetic stimulation has an activity-dependent suppressive effect. $J$. Neurosci., 32: 12361-12365, 2012.

Plewnia C., Zwissler B., Längst I., Maurer B., Giel K., Krüger R. Effects of transcranial direct current stimulation (tDCS) on executive functions: Influence of COMT Val/Met polymorphism. Cortex, 49: 1801-1807, 2013.

Reato D., Rahman A., Bikson M., Parra L.C. Effects of weak transcranial alternating current stimula- 
tion on brain activity. A review of known mechanisms from animal studies. Front. Hum. Neurosci., 23: 687, 2013.

Reis J., Swayne O.B., Vandermeeren Y., Camus M., Dimyan M.A., Harris-Love M., Perez M.A., Ragert P., Rothwell J.C., Cohen L.G. Contribution of transcranial magnetic stimulation to the understanding of cortical mechanisms involved in motor control. J. Physiol. (Lond.), 586: 325-351, 2008.

Reis J., Schambra H.M., Cohen L.G., Buch E.R., Fritsch B., Zarahn E., Celnik P.A., Krakauer J.W. Noninvasive cortical stimulation enhances motor skill acquisition over multiple days through an effect on consolidation. Proc. Natl. Acad. Sci. USA, 106: 1590-1595, 2009.

Ridding M.C. and Ziemann U. Determinants of the induction of cortical plasticity by non-invasive brain stimulation in healthy subjects. J. Physiol., 588 (Pt 13): 2291e304, 2010.

Riecker A., Gröschel K., Ackermann H., Schnaudigel S., Kassubek J., Kastrup A. The role of the unaffected hemisphere in motor recovery after stroke. Hum. Brain Mapp., 31: 1017-1029, 2010.

Sandrini M. and Cohen L.G. Noninvasive brain stimulation in neurorehabilitation. Handb. Clin. Neurol., 116: 499-524, 2013.

Sandrini M., Umilta C., Rusconi E. The use of transcranial magnetic stimulation in cognitive neuroscience: a new synthesis of methodological issues. Neurosci. Biobehav. Rev., 35: 516-536, 2011.

Schaechter J.D. Motor rehabilitation and brain plasticity after hemiparetic stroke. Prog. Neurobiol., 73: 61-72, 2004.

Seniów J., Bilik M., Lesniak M., Waldowski K., Iwanski S., Czlonkowska A. Transcranial magnetic stimulation combined with physiotherapy in rehabilitation of poststroke hemiparesis: a randomized, double-blind, placebo-controlled study. Neurorehabil. Neural Repair, 26: 1072-1079, 2012.

Schambra H.M., Abe M., Luckenbaugh D.A., Reis J., Krakauer J.W., Cohen L.G. Probing for hemispheric specialization for motor skill learning: a transcranial direct current stimulation study. $J$. Neurophysiol., 106: 652-661, 2011.

Stefan K., Kunesch E., Cohen L.G., Benecke R., Classen J. Induction of plasticity in the human motor cortex by paired associative stimulation. Brain, 123: 572-584, 2000.

Stinear C.M., Barber P.A., Smale P.R., Coxon J.P., Fleming M.K., Byblow W.D. Functional potential in chronic stroke patients depends on corticospinal tract integrity. Brain, 130: 170-180, 2007.
Swayne O.B., Rothwell J.C., Ward N.S., Greenwood R.J. Stages of motor output reorganization after hemispheric stroke suggested by longitudinal studies of cortical physiology. Cerebr. Cortex, 18: 1909-1922, 2008.

Takeuchi N., Chuma T., Matsuo Y., Watanabe I., Ikoma K. Repetitive transcranial magnetic stimulation of contralesional primarymotor cortex improves hand function after stroke. Stroke, 36: 2681-2686, 2005.

Takeuchi N., Tada T., Toshima M., Matsuo Y., Ikoma K. Repetitive transcranial magnetic stimulation over bilateral hemispheres enhances motor function and training effect of paretic hand in patients after stroke. J. Rehabil. Med., 41: 10491054, 2009.

Takeuchi N. and Izumi S.I. Noninvasive brain stimulation for motor recovery after stroke: mechanisms and future views. Stroke Res. Treat., 584727: 1-10, 2012a.

Takeuchi N. and Izumi S.I. Maladaptive plasticity for motor recovery after stroke: mechanisms and approaches, Neural Plast., 359728: 1-9, 2012 b.

Takeuchi N. and Ikoma K. 1 Hz rTMS over unaffected hemisphere in stroke patients alters bilateral movements and coupling betweenmotor areas. Clin. Neurophysiol., 121 (Suppl. 1): s316, 2010.

Talelli P., Cheeran B.J., Teo J.T., Rothwell J.C. Pattern-specific role of the current orientation used to deliver Theta Burst Stimulation. Clin. Neurophysiol., 118: 1815e23, 2007.

Talelli P., Greenwood R.J., Rothwell J.C. Arm function after stroke: neurophysiological correlates and recovery mechanisms assessed by transcranial magnetic stimulation. Clin. Neurophysiol., 117: 1641-1659, 2006.

Terney D., Chaieb L., Moliadze V., Antal A., Paulus W. Increasing human brain excitability by transcranial high-frequency random noise stimulation. J. Neurosci., 28: 14147e55, 2008.

Utz K.S., Dimova V., Oppenlander K., Kerkhoff G. Electrified minds: transcranial direct current stimulation (tDCS) and galvanic vestibular stimulation (GVS) as methods of non-invasive brain stimulation in neuropsychology. A review of current data and future implications. Neuropsychologia, 48 : 2789-2810, 2010.

Ward N.S. and Cohen L.G. Mechanisms underlying recovery of motor function after stroke. Arch. Neurol., 61: 1844-1848, 2004.

Wang H.Y., Crupi D., Liu J., Stucky A., Cruciata G., Di Rocco A., Friedman E., Quartarone A., Ghilardi 
M.F. Repetitive transcranial magnetic stimulation enhances BDNF-TrkB signaling in both brain and lymphocyte. J. Neurosci., 31: 11044-11054, 2011.

Wolters A., Sandbrink F., Schlottmann A., Kunesch E., Stefan K., Cohen L.G., Benecke R., Classen J. A temporally asymmetric Hebbian rule governing plasticity in the human motor cortex. $J$. Neurophysiol., 89: 2339-2345, 2003.
Yoon K.J., Lee Y.T., Han T.R. Mechanism of functional recovery after repetitive transcranial magnetic stimulation (rTMS) in the subacute cerebral ischemic rat model: neural plasticity or anti-apoptosis? Exp. Brain Res., 214: 549-556, 2011.

Ziemann U., Rothwell J.C., Ridding M.C. Interaction between intracortical inhibition and facilitation in human motor cortex. J. Physiol. (Lond.), 496: 873-881, 1996. 